\title{
Quantum Wave Mixing and Probing of Photonic States in 1D space
}

\author{
T. Hönigl-Decrinis ${ }^{1}$, A.Yu. Dmitriev ${ }^{2}$, R. Shaikhaidarov ${ }^{1,2}$, \\ $\underline{\text { V.N. Antonov }}^{1,3, *}$, O.V. Astafiev ${ }^{1,2}$ \\ ${ }^{1}$ Physics Department, Royal Holloway, University of London \\ ${ }^{2}$ Moscow Institute of Physics and Technology \\ ${ }^{3}$ Skolkovo Institute of Science and Technology \\ *E-mail:v.antonov@skoltech.ru
}

DOI:10.31868/RFL2018.28-29

The wave mixing is well revealed and theoretically described phenomenon of a nonlinear optics. It has applications in phase conjugation, generation of squeezed states, parametric frequency conversion, signal regeneration schemes and exploited significantly for spectroscopic study of various systems. The wave mixing was thoroughly investigated in a medium such as fibre, atomic beams and vapours, with various number of mixed waves, exploiting two or more levels of a system. However, any medium represents a huge ensemble of atoms, so one needs many photons to drive the medium efficiently. Also, energy levels are broadened in homogeneously and hence what is accessible in wave mixing experiment is collective response of an ensemble of atoms. Quantum Wave Mixing (QWM) reveals itself as an elastic scattering of coherent classical and non-classical photonic states of electromagnetic waves on a single atom. We show a spectrum, corresponding to four-wave mixing of non-classical photonic states with a fingerprint of interacting photon states: the number of frequency peaks due to stimulated emission
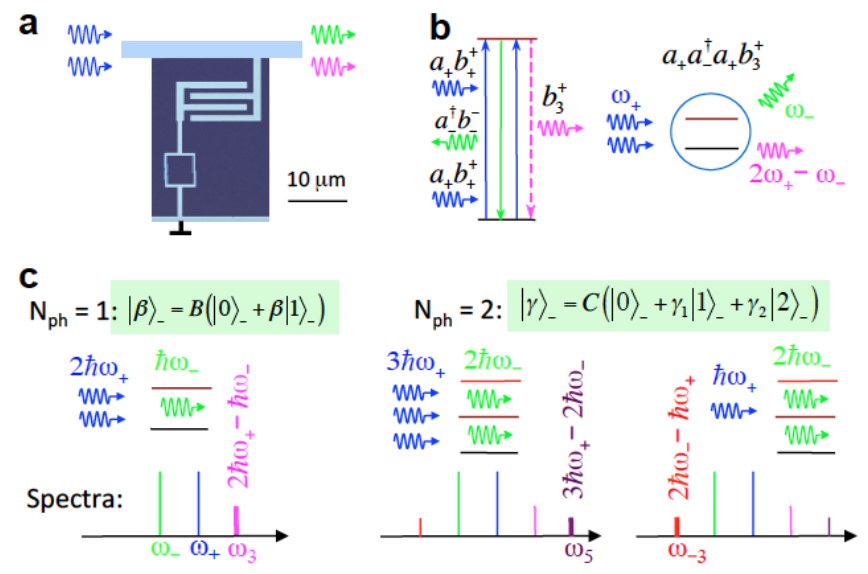

Fig.1. a) A false colored SEM image of the device: a superconducting loop with four Josephson junctions, behaving as an artificial atom, is embedded into a transmission line and strongly interacts with propagating electromagnetic waves. b) Four-wave mixing processes resulting in the single-photon field creation at ${ }_{3}=2_{+}$. In classical mixing, the process operators $a_{+} a^{*} a_{+} b_{3}^{+}$comes in pair with the symmetric one $a a_{+}^{*} a b_{3}^{+}$. In the mixing with non-classical states, the time symmetry is broken resulting in the asymmetric spectrum. c) Schematic representation of QWM with non-classical coherent states and sensing of the coherent quantum states. Two sequential pulses and then + are applied breaking time symmetry and, therefore, spectrum symmetry. Coherent photonic states are created in the atom by the first pulse at and then mixed with the second pulse of ${ }_{+}$. Single-photon, $N_{\mathrm{ph}}=1$, state $\mid>$ can only create a peak at ${ }_{3}=2+$ because only one photon at can be emitted from the atom. Two 
photon, $N_{\mathrm{ph}}=2$, coherent state $\mid>$ results in creation of an additional peak at $3+2$, because not more than two photons can be emitted. Also one photon of can be absorbed, $N_{\mathrm{ph}}-1$, creating additional left-hand-side peak at 2

always exceeds by one the ones due to absorption, see Fig.1. We also study four- and higher-order wave mixing of classical coherent waves. In this case the time dynamics of the peaks exhibits a series of Bessel-function quantum oscillations with orders determined by the number of interacting photons [1].

In our study we operate in the microwave range of electromagnetic radiation. The two level superconducting circuit, qubit, serves as the artificial atom which scatter the microwave radiation, see Fig. 1. In a wider context these artificial atoms may be a building blocks of novel on-chip quantum electronics, which utilize the quantum nature of electromagnetic waves [2].

\section{References}

[1] A Dmitriev et al, Nature Comm., 8, 1352 (2017)

[2] O. Astafiev et al., Nature, 449, 588-590 (2007). 